\title{
Medicina y Salud
}

In Cres. Vol. $4 N^{\circ} 1:$ pp. 75-91, 2013

\section{CONSTRUCCIÓN DE LA SEXUALIDAD EN EDAD REPRODUCTIVA CON ENFOQUE DE GÉNERO DESDE LA PERSPECTIVA MÉDICA. Bases sociohistórico culturales*}

\author{
CONSTRUCTION OF THE SEXUALITY IN REPRODUCTIVE AGE WITH \\ APPROACH OF GENERE FROM THE MEDICAL PERSPECTIVE. \\ Social historical cultural bases
}

\author{
Walter Obeso Terrones ${ }^{1}$ \\ Moisés Barrantes Cabrera
}

\section{RESUMEN}

Esta investigación, de tipo cualitativo, con perspectiva dialéctica y de género, tuvo los siguientes objetivos: a) Explicar el proceso de construcción de la sexualidad en edad reproductiva; b) Analizar las semejanzas y diferencias de la construcción de la sexualidad; y c) Determinar las bases sociohistórico y culturales actuales en que se construye la sexualidad en edad reproductiva con enfoque de género y desde la perspectiva médica. Material y métodos: Mediante entrevista profunda a un total de 24 unidades de análisis 18 adultos jóvenes (ocho varones y diez mujeres) y 6 médicos docentes varones; se utilizó el método hermenéutico de la historia-oral temática, apoyado por la entrevista semiestructurada como técnica. Se obtuvo información según su utilidad, hasta cumplir el principio de saturación o "bola de nieve"; es decir, hasta que se repita la información por parte de los sujetos en estudio. Resultados: Se encontraron tres categorías como aporte a los estudios de la sexualidad que son causa de los problemas de salud pública en el género femenino en el Perú, que han definido las conclusiones: 1) La sexualidad se construye con fuerte influencia de padres, familiares, educadores y medios de comunicación, que interactúan en el contexto de las personas; 2) La sexualidad humana ocurre dentro del contexto socialcultural del machismo dominante acep-

* Recibido: 25 de mayo del 2013; aprobado: 30 de mayo del 2013.

1 Doctorando en Medicina de la Escuela de Post Grado de la Universidad Nacional de Trujillo. Artículo extraído de la Tesis del mismo nombre, para obtener el grado de Doctor en Medicina. E-mail: wobesot@gmail.com

2 Médico Asesor de la mencionada tesis. E-mail: moisesbarrantesc@hotmail.com 
tado por la sociedad, lo que produce diferencias y discriminación entre los géneros; 3) La sexualidad humana reposa en las bases sociohistórico y culturales de cada sociedad, de cada comunidad, en cada hogar, sobre las que influyen las normas y reglas impuestas por la misma sociedad.

PALABRAS CLAVE: Sexualidad, género, cultura, sociedad.

\section{ABSTRACT}

This research, of qualitative type, with dialectical perspective of genre, had the following objectives: a) To explain the process of construction of the sexuality in reproductive age; b) To analyze the similarities and differences of the construction of the sexuality; and c) To determine the social historical and cultural bases current in that the sexuality is constructed in reproductive age by approach of genre and from the medical perspective. Material and methods: By means of the deep interview technic, a total of 24 units of analyses adult 18 young (eight males and ten women) and 6 medical educational males; we use the hermeneutic method of the oral thematic history, supported by the interview semi structured like technical; information was obtained according to his utility, up to fulfilling the saturation principle or " ball of snow "; it is to say until the information should repeat itself on the part of the subjects in study. Results: We found three categories as contribution to the studies of the sexuality that are a reason of the problems of public health in the feminine genre in Peru, which our Conclusions have defined: 1) The sexuality constructs itself with fort influence of parents, relatives, educators and mass media that interact in the context of the persons; 2) The human sexuality happens inside the social cultural context of the dominant machismo accepted by this society, which produces differences and discrimination among the genres; 3 ) The human sexuality historical social rests in the bases and cultural of every society, of every community, in every home, on that they influence the procedure and rules imposed by the same society.

KEY WORDS: Sexuality, genre, culture, society.

\section{INTRODUCCIÓN}

La sexualidad constituye un aspecto inherente a los seres humanos durante todo el transcurso de su vida, desde el momento de la concepción hasta la muerte; es una "forma de expresión integral de los seres humanos, vinculada a los procesos biológicos, psicológicos y sociales del sexo". ${ }^{1}$ Como fuente de placer y de bienestar es un elemento enriquecedor en lo personal, con honda repercusión en lo familiar y en lo social. ${ }^{1,2}$ Por todo ello, el derecho al disfrute de la sexualidad, en la multiplicidad de su potencialidad, es un derecho humano inalienable, como el derecho a la vida, a la libertad, a la equidad y a la justicia social, así como a la salud, a la educación y al trabajo, entre otros. ${ }^{3}$

A diferencia del sexo, la sexualidad no es innata sino formada y depende 
de las costumbres, épocas y países; es cambiante y dinámica y su función no es sólo la reproducción de la especie sino la búsqueda del intercambio, la creatividad y el placer. ${ }^{16}$ Es un proceso de aprendizaje, que se inicia desde el nacimiento, en el cual, intervienen la familia, la escuela, la sociedad, los medios de comunicación y todo el ambiente. ${ }^{11}$

La sexualidad es el elemento organizador de la identidad total de las personas. Este proceso cobra significación capital en la adolescencia, donde el logro de tal identidad se considera el objetivo central; razón por la cual, esta etapa de la vida es el momento privilegiado para la educación en salud y evitar consecuencias en contra de la salud física, psicológica y de la calidad de vida en los jóvenes. ${ }^{5,7}$ La relación entre las condiciones de vida de la mujer vinculadas a su desempeño del rol de género y a su salud, dentro de un ambiente predominantemente machista, con violencia basada en género, enfermedades obtenidas por contagio sexual y ruptura de vínculos matrimoniales con destrucción de vínculos familiares han sido objeto de estudio y de trabajo permanentes por parte de médicos, psicólogos, sociólogos y antropólogos ${ }^{4}$; y defendidos por movimientos sociales, como el movimiento feminista y grupos alternativos de salud de la mujer. ${ }^{21}$ Sin duda, el hogar, la escuela, los medios de comunicación y la sociedad, en su conjunto, todavía aportan rígidas representaciones de lo que es "ser varón" y "ser mujer", las cuales van estructurando el rol de género desde el momento mismo de la concepción y llevan implícitos criterios francamente discriminatorios entre ambos géneros. ${ }^{8,9}$

Las investigaciones médicas en salud sexual y reproductiva se han desarrolladas por más de cuatro décadas en el Perú; sin embargo, muchos de los problemas estudiados aún existen o se han incrementado; y los resultados de sus programas o actividades, satisfactorios o no, han quedado en suspenso. En ese sentido, se parte de la premisa que hay graves problemas de salud, especialmente, en el área de la salud sexual y reproductiva de la mujer de todo tipo, al parecer, como consecuencia de no asumir una educación sexual responsable dentro de la sociedad y cuyos antecedentes se sintetizan a continuación, argumentando la problematización.

La mortalidad materna y perinatal: En el 2008, la tasa de mortalidad materna fue de 100 por cada 100 mil nacidos vivos (por edades extremas, intervalos intergenésicos cortos, nivel educativo bajo, falta de cuidado del embarazo e inadecuada atención del parto institucional, entre otras) y la tasa de mortalidad perinatal fue de 23 defunciones por mil embarazos de siete o más meses. ${ }^{23} \mathrm{El}$ estado se preocupa por recuperar la salud orgánica de muchas mujeres y niños, pero es incapaz de prever que los casos se repitan exponencialmente de acuerdo 
al ritmo de crecimiento poblacional, lo que origina gastos millonarios innecesarios por encima de sus posibilidades de atención a futuras generaciones. ${ }^{23}$

Aborto: Muchos de los abortos son producidos como consecuencia de violaciones a mujeres. ${ }^{1}$

Infecciones de transmisión sexual (ITS/VIH/SIDA: infecciones de diversa etiología, cuya forma de contagio ocurre a través de las relaciones sexuales sin protección. Aunque el VIH se puede transmitir por otras vías, la transmisión sexual es altamente predominante (96\% en el Perú). ${ }^{23}$

Cáncer de cuello uterino y de mama: son los cánceres más frecuentes en las mujeres peruanas $\mathrm{y}$, al igual que el cáncer de mama, puede ser detectado tempranamente durante la atención de salud reproductiva, así como con la práctica del autocuidado. ${ }^{24}$

Violencia contra la mujer: la violencia basada en el género (VBG) abarca muchos tipos de comportamientos físicos, emocionales y sexuales, nocivos para las mujeres y las niñas, que son practicados con más frecuencia por miembros de la familia; pero a veces también por extraños. ${ }^{10} \mathrm{La}$ VBG es tan frecuente que ocurre en más del $50 \%$ de las mujeres peruanas en edad reproductiva $;{ }^{23}$ la mortalidad materna ha sido calificada por la Organización Panamericana de Salud (OPS), como el reflejo más claro de la discriminación y el bajo estatus social de las mujeres y es reconocida como un marcador de compromiso de los Estados con el derecho a la salud. ${ }^{7}$ En general, es un problema que obedece a múltiples causas, influido por factores sociales, económicos, psicológicos, jurídicos, culturales y biológicos, los cuales, más del $75 \%$ de los casos, lo sufren las mujeres. ${ }^{27}$

Ante este panorama se piensa que miles de mujeres y niñas, que corresponden al 50\% de la población peruana, están en peligro de tener baja calidad de vida, sufrir severas enfermedades y hasta la muerte, por desconocimiento de una correcta y eficiente educación sexual.

En el sector salud, no existen estudios regionales ni locales sobre sexualidad con abordaje de género. Algunos trabajos existentes tienen enfoque biomédico y todos o casi todos están enfocados en estudiar la recuperación de la salud y sus consecuencias físicas, pero no se orientan a esbozar medidas de solución alternativas que vayan a combatir la raíz misma del problema.

\section{JUSTIFICACIÓN}

La investigación servirá de referente para mejorar políticas públicas en salud, ${ }^{13}$ programas médicos nacionales de salud sexual y reproductiva que 
actualmente se dan con muy débil proyección social. Servirá también para resaltar y conocer los derechos humanos sexuales y reproductivos de hombres y mujeres; mejorar los bajos niveles de conocimiento de prevención contra daños físicos y sicológicos en la esfera sexual y recuperarse de la ausencia de cultura de equidad de género, pues pensamos que la educación para una sexualidad responsable evita problemas profundos en la sociedad.

\section{PROBLEMA}

¿Cómo es la construcción de la sexualidad con enfoque de género, en la edad reproductiva, desde la perspectiva médica? Trujillo, enero a diciembre 2012.

\section{OBJETIVOS}

a) Explicar el proceso de construcción de la sexualidad en edad reproductiva con enfoque de género desde la perspectiva médica;

b) Analizar las semejanzas y diferencias de la construcción de la sexualidad, con enfoque de género desde la perspectiva médica, entre varones y mujeres en edad reproductiva;

c) Determinar las bases sociohistórico y culturales vigentes en las que se construye la sexualidad en edad reproductiva, con enfoque de género, desde la perspectiva médica.

\section{MATERIAL Y MÉTODOS}

El estudio se llevó a cabo en centros de atención del Ministerio de Salud, de los distritos de Víctor Larco Herrera, Huanchaco y Trujillo. Se utilizó el abordaje de la investigación cualitativa interpretativa mediante el método hermenéutico, con perspectiva dialéctica y de género, teniendo como base la necesidad de describir, analizar e interpretar los planteamientos que direccionan este estudio ${ }^{6}$ y de formular explicaciones teóricas confiables, basadas en filósofos y teóricos expertos investigadores de género y sexualidad humana. Al hablar de la dialéctica utilizamos los principios de historicidad, totalidad y contradicción. ${ }^{12}$

La población y muestra en estudio estuvieron conformadas por 24 unidades de análisis: 18 adultos jóvenes (ocho varones, en adelante designados con los números del 1 al 8 para este estudio y 10 mujeres en adelante designadas con los números del 9 al 18), que reunieron los requisitos de inclusión: casado o conviviente, de 30 a 50 años, con hijo(s), aparentemente sanos, con secundaria completa y de las categorías socioeconómicas A, B, D y E; el nivel socio económico (NSE) se define como la capacidad para acceder a un conjunto de bienes y 
estilo de vida, entre ellos: grado de instrucción, posesión de bienes, hacinamiento. El NSE D tiene ingresos económicos mensual por integrante de no menos de $S / .1,030.00$ y el NSE E, por lo menos S/.730.00. Para efecto de la complementación de información sobre la perspectiva médica, se entrevistó a seis médicos docentes de facultades de medicina de universidades de Trujillo, según requisitos de inclusión (casado, de 30 a 50 años, con hijo(s), que hagan docencia universitaria y pertenecientes a las clases socioeconómicas " $\mathrm{A}$ ", por tener un ingreso económico mensual por integrante en el hogar no menor a $\mathrm{S} / .10,720.00$, y B con un ingreso mensual de $S / .2,990.00$. y que acudieron a consultar, acompañando algún familiar a los centros de salud mencionados líneas arriba. Se utilizó el método de la Historia-Oral Temática, apoyado por la técnica de la entrevista semiestructurada ${ }^{(16)}$, realizada previa firma del Acta de Consentimiento Informado correspondiente. Se obtuvo información por eficacia, cumpliendo el principio de saturación o "bola de nieve"; es decir, hasta que se repita la información por parte de los sujetos en estudio. Para la interpretación de las respuestas se consideró conveniente trabajar con la triangulación metodológica; es decir, tomar múltiples puntos de referencia para localizar una posición desconocida. ${ }^{27,28}$ Para el análisis de datos se trabajó con herramientas, como realizar una reflexión crítica sobre las condiciones de producción y aprehensión del significado de los argumentos expresados; y comprender el modo de funcionamiento del discurso, los principios de su organización y las formas de producción social del sentido de las palabras que los sujetos involucrados dieron a una determinada cuestión. ${ }^{5}$ Aplicar el análisis de discurso implica comprender e interpretar el sentido del discurso; sin querer descubrir nada nuevo ni pretender arribar a verdades absolutas, sino que apenas se hará una nueva interpretación y relectura de la realidad. ${ }^{16}$

\section{RESULTADOS}

En la pre configuración de la realidad descrita en las primeras entrevistas, se logró las pre categorías simples que permitieran descubrir, reservadamente, las partes que forman el entramado de esa realidad sociocultural, en cuanto a la formación de su sexualidad durante sus vidas, que son:

a) Diecisiete de todos los participantes (más del 70\%) identificaron a la comunicación como importante para la formación de su sexualidad durante la niñez. Lamentablemente esto no se produce entre sus familiares.

b) Su autoformación en el conocimiento sobre su sexualidad, aborto, violencia física e infidelidad conyugal. 
c) La tercera precategoría o tema recurrente que expresan con ansiedad los participantes, al responder, es sobre el placer sexual y su relación con el bienestar de la mujer, del hombre y la familia.

Seis de las diez mujeres (60\%) expresaron que no eran felices en su hogar por maltrato y agresión física de sus esposos y porque sus relaciones sexuales no eran satisfactorias. Sólo una de ellas (10\%) dijo que sí era muy feliz con su marido, porque "tenemos relaciones sexuales muy satisfactorias y no voy a dejar que mi esposo se vaya con otra mujer; por eso, trato de complacerlo...”. Seis (70\%) de los varones opinaron que tampoco eran felices con su pareja porque estaban ya casados varios años (algunos más de doce años); otros, porque ya no les "gustaba" su mujer; otros, porque tenían mucho trabajo. Asimismo, el $80 \%$ de estos varones opinaron que sí buscaban otra mujer eventualmente y que les parecía natural que así sea, porque "cada día uno tiene que trabajar mucho y tengo que buscar alguna diversión...”. Otras opiniones fueron: “...no maltrato a mi mujer, excepto si me da cólera porque deja solos a mis hijos en casa y se va conversar con sus amigas...”; “...uno como hombre puede tener otras mujeres para buscar el placer...; la mujer no puede ser así, porque entonces sería una mujer de la calle...".

En relación al contexto sociocultural, en el que las personas construyen su sexualidad, se ha encontrado tres precategorías determinantes de ello:

a) Las mujeres actúan con predominio de sus emociones y sentimientos; y los hombres son vistos como más fuertes, con poder y dominio de la situación sexual;

b) Machismo dominante aceptado socialmente;

c) Discriminación y violencia basada en género.

Se realizó la triangulación, con el objetivo de incrementar la validez de los resultados de la investigación, mediante la depuración de las deficiencias intrínsecas de usar un solo método de recolección de datos y para controlar el sesgo personal del investigador. De este modo, puede decirse que cuanto mayor es el grado de triangulación, mayor es la fiabilidad de las conclusiones alcanzadas. ${ }^{27,28}$ Con ese propósito se formularon las mismas preguntas, al entrevistar a médicos docentes: uno de ellos del NSE A y cinco del NSE B.

La triangulación de los primeros resultados de la entrevista semiestructurada, con los adultos jóvenes, varones y mujeres, y con los médicos docentes de facultades de medicina en universidades, reveló que la construcción de la sexualidad humana en edad reproductiva con enfoque de género es un fenómeno que 
se construye de acuerdo a la cultura de cada pueblo y hogar dentro de las normas y reglas impuestas por la sociedad, la misma que dio lugar a la primera categoría de análisis y discusión de la tesis:

I. Construcción de la sexualidad humana según la cultura de cada sociedad, de cada hogar, con respecto a las normas y reglas impuestas por la misma sociedad. Con sus temas:

a) Comunicación en la familia,

b) La autoformación sexual del individuo.

c) Infidelidad, aborto y violencia física y psicológica contra la mujer.

d) Sexualidad, placer y bienestar.

La segunda categoría de análisis, preconfigurada sobre el resultado de las entrevistas de los informantes, fue resultado de preguntar acerca de las diferencias y semejanzas en la construcción de la sexualidad humana según género. Las respuestas de los adultos jóvenes de ambos sexos que asistieron al centro de salud revelaron:

II. Construcción de la sexualidad humana ocurre en el contexto socialcultural del machismo dominante aceptado, lo que produce diferencias, discriminación y consecuencias físicas, psicológicas y sociales entre los géneros, sean profesionales o no. Con sus temas y sub temas:

a) Contexto sociocultural de la construcción de la sexualidad:

- Las mujeres actúan con predominio de sus emociones y sentimientos; los hombres son vistos como más fuertes, con poder y dominio de la situación sexual.

- Machismo dominante aceptado socialmente.

- Discriminación y violencia basada en género.

b) Semejanzas están dadas, porque ambos son seres humanos complementarios y comparten responsabilidades del hogar.

Una tercera categoría, que pareciera asomarse como fundamental dentro el estudio, es lo que respecta a la influencia de la cultura y la sociedad en la que se desenvuelven los seres humanos. Ello daría lugar a:

III. Analizar la influencia positiva sociocultural actual vs. influencia negativa en la construcción de la sexualidad de la sociedad. Con sus temas y sub temas:

a) Influencia positiva:

- De la ciencia, la tecnología y las nuevas tecnologías de información y comunicación (NTIC). 
- Conservación de valores en algunos sectores de la sociedad y el Estado.

\section{b) Influencia negativa:}

- Las NTIC.

- Sexualidad humana considerada mala y prohibida por la sociedad.

- Familia relega a la mujer y favorece al varón.

- Escasa educación sexual como política educacional del Estado.

- Pérdida de valores en la sociedad.

\section{DISCUSIÓN}

La investigación sobre construcción de la sexualidad humana gira en torno a todo el conjunto de legados axiológicos, normas, creencias y actitudes que se han transmitido de generación en generación, mediante la comunicación de padres a hijos, o de abuelos a sus descendientes, agrupados dentro de una familia que, como muchas otras, conforman la sociedad, constituyendo la compleja red de representaciones sobre la vivencia de la sexualidad. Lo encontrado está de acuerdo, primariamente, con lo encontrado por otros autores ${ }^{1,8}$ La historia de la humanidad ha cambiado mucho la concepción de la sexualidad humana. El enfoque social ha variado por la cultura, fundamentalmente, desde la perspectiva social, de acuerdo con la aceptación o no que cada cultura tiene o "debe tener", según los cánones socialmente aceptados hacia su propia sexualidad. ${ }^{21}$

Si bien las interacciones sexuales son una realidad fundamental de las relaciones sociales. ${ }^{3}$ las experiencias reales de la sexualidad de las niñas y las mujeres están determinadas por una serie de permisos y restricciones que, rara vez, son aplicados por igual a los niños y los hombres. ${ }^{25}$ Con frecuencia, los intentos de las mujeres por afirmar su autonomía sexual chocan con la oposición de la familia, la sociedad y el Estado, que tratan de controlarla y que muchas veces se trata de una relación en la que las niñas y las mujeres dependen materialmente de los hombres. ${ }^{19}$ Esto es lo que se ha establecido como aporte al estudio de la sexualidad femenina, que a pesar de la esencia comunicativa como seres humanos, parece que cuesta trabajo día a día mantener una comunicación abierta y sincera con la pareja, lo que está de acuerdo con lo encontrado por Ortiz. ${ }^{26}$

Un alto número de los participantes identificaron a la comunicación como importante para la formación de su sexualidad durante la niñez, que no se produce entre sus familiares. Más del $60 \%$ de los participantes (15 de ellos) cree que la religión fue un instrumento que impidió hablar de conductas sexuales en 
la niñez con sus padres, más notorio, en los niveles socioeconómicos D y E, pero también muy notorio en niveles más altos, cuando uno de los profesionales declaró que "vengo de una familia muy creyente y mis padres no hablaban nunca del tema sexual".

La falta de comunicación provoca sentimientos de culpa en la mujer y de inseguridad sobre la virilidad en el hombre. Esto ha causado que el $55 \%$ de la población mayor de 40 años de edad sufra algún tipo de frustración en las mujeres y falta de erección o baja cuenta espermática en el hombre, según De los Ríos. ${ }^{11}$

Una precategoría simple que se consolidó en los estudios finales: la autoformación en el conocimiento sobre la sexualidad, tanto en hombres como en mujeres. Las respuestas, en el $90 \%$ de los casos, del sexo masculino reconocen que nunca o casi nunca tuvieron educación sexual en su hogar, en el colegio o en la universidad (caso de los médicos). Hombres y mujeres consideran que la infidelidad masculina es frecuente y mucho menor en la mujer, siendo uno de los principales problemas en los hogares con todas las secuelas que ella trae. La familia, célula básica de la sociedad, por lo general, se trastorna intensamente con la infidelidad; 70\% de las mujeres declaró que, al menos una vez, sus esposos han dado indicios de que fueron infieles. Entre los entrevistados se tolera la creencia que la infidelidad masculina es menos grave que la femenina. Se piensa que los hombres son infieles de forma natural o "biológica" y ello se "aprueba" en el $80 \%$ de varones y el $100 \%$ de las mujeres; sin embargo, se ve como una falta muy grave y no es admitida por la sociedad que la mujer sea infiel ( $90 \%$ de las mujeres niega haber sido alguna vez infieles a su pareja, y $80 \%$ afirman que nunca lo serían).

Otro tema recurrente que expresa con ansiedad, al responder los participantes, es sobre el placer sexual y su relación con el bienestar de la mujer, del hombre y la familia. La dimensión placentera de la sexualidad es el centro de temores, represiones y tabúes. La raíz de muchos problemas en la vida de la pareja se hunde en este terreno oscuro que convierte al placer amoroso en una experiencia de culpa y vergüenza, por obra de los prejuicios y la ignorancia o desorientación de la sociedad. ${ }^{22}$

En esta investigación se encontró que seis de las diez mujeres (60\%) expresaron que no eran felices en su hogar, por el maltrato y agresión física de sus esposos y porque sus relaciones sexuales no eran satisfactorias. Y seis (70\%) de los varones de la muestra opinaron que no eran felices con su pareja debido a su estado de convivencia de muchos años (algunos más de doce años); 
algunos, porque ya no les "gustaba" su mujer; otros, porque tenían mucho trabajo. El $80 \%$ de los varones opinaron que sí buscaban otra mujer eventualmente y que les parecía natural que así sea, porque "uno tiene que trabajar mucho y tengo que divertirme...”.

En general, los varones piensan que la mujer es un complemento del hombre, en el sentido que debe servirlo en su casa, servir a sus hijos y en lo sexual, lo que es calificado por varios de los varones de la muestra como "una buena mujer". Esto ocurre en los NSE D y E; sin embargo, en los NSE A y B, cuando se entrevistó a los médicos, el concepto de una "buena mujer" es muy diferente, y se argumenta que la mujer es el complemento del hombre porque debe tener los mismos ideales, los mismos sentimientos y las mismas oportunidades de trabajo para su progreso personal y espiritual, y que sea una mejor madre de familia. Aunque, entre líneas se puede leer que hay cierto grado de machismo, cuatro de ellos (70\%) opinaron que la mujer debe encargarse de criar a los hijos en los primeros años; aunque posteriormente la crianza y educación sea responsabilidad de ambos. Igualmente, se prefiere que la mujer consiga un trabajo "que no la aleje muchas horas del hogar, ni mucho menos por las noches..." y también "...mi mujer es mía y debe amarme tanto como yo la amo y la respeto; por eso tenemos un hogar sólido...". En el ámbito religioso, los padres (40\% de las madres y $40 \%$ de los padres) piden y exigen que sus hijos asistan a misa por lo menos los días domingo.

En el ámbito social: en la época de ir al colegio, si bien asisten todos a colegios mixtos, las actividades en el recreo son muy diferentes para hombres o para mujeres. Tal vez, esta es la época en que las niñas tienen mayor opción a incrementar su libertad. Los padres manifiestan que sus hijas salen de su casa "solas y no sabemos si van o no al colegio" ${ }^{17}$ esta situación de falta de control por parte de los padres con sus hijos, permite que en la ciudad se oferten fiestas "juveniles" para adolescentes al mediodía y por las tardes, con asistencia masiva de escolares con todos los peligros de por medio y, principalmente, de relaciones sexuales, uso de drogas, alcohol y embarazos no deseados, sin nombrar las enfermedades de transmisión sexual (ETS), como el VIH y enfermedades venéreas, dentro de las más comunes, con todas sus consecuencias derivadas, como abortos, embarazos de adolescentes con todos sus riesgos, ETS, violencia juvenil, violencia basada en género, etc. Con el alcohol, se desencadena la violencia entre varones y de los varones en contra de sus "enamoradas", repitiendo muchas veces el modelo de comportamiento que cuando niño vio en su hogar. Así, con los problemas derivados de esta situación social, el Estado hará fuertes 
desembolsos de dinero para recuperar la salud de algunos jóvenes y, en otros casos, los perderá definitivamente; presupuestos que cada año se van incrementando sin hacer nada por atacar las causas estructurales que son sociales y culturales, establecidos en esta investigación, de esa grave realidad de la salud pública del Perú.

La construcción de la sexualidad humana ocurre en el contexto social cultural del machismo dominante aceptado, lo que produce diferencias, discriminación y consecuencias físicas, psicológicas y sociales entre los géneros, sean profesionales o no.

Muchos de los participantes (75\%) expresaron que los hombres y las mujeres tienen diferencias en la manera de expresar los sentimientos, pues las mujeres ven el pasado con nostalgia y los hombres se sienten muy cómodos en el presente y se divierten y gozan más de la vida (refieren que "ellos" salen con sus amigos, llegan tarde a la casa, salen solos, toman licor casi todos los fines de semana y aún entre semana, manejan el dinero, etc.). En cuanto a la forma de expresar los sentimientos, la diferencia radica en la forma de manifestarla. Expresar los sentimientos se sigue considerando un signo de debilidad tanto en mujeres (50\%) como en hombres (80\% de la muestra de NSE D y E y en el $30 \%$ de los NSE A y B). También se estableció que se acepta que existen emociones tradicionalmente masculinas y otras femeninas; por ejemplo, la mujer es más libre para expresar la tristeza y los hombres la cólera o enfado. Los hombres manifiestan estar en calma más a menudo; sin embargo, las mujeres se ponen nerviosas con más facilidad (70\% de las mujeres NSE D y E aceptan ser muy nerviosas). Lamentablemente, muchos factores, como el grado de educación, los padres no siempre son conscientes de la trascendencia que reviste atender, integrar y conducir las emociones infantiles sin discriminar entre hijos varones o mujeres. En la vida de pareja, se ha comprobado que la estabilidad de la relación y el éxito en la toma de decisiones dependen mucho de la madurez y estabilidad emocional de sus miembros.

En relación a la construcción de la sexualidad dentro del contexto socialcultural, se encontró que existe discriminación por género. Se conoce como discriminación al acto de separación de una persona o grupo de personas a partir de criterios determinados. Creerse superior a otra persona y así, maltratarle física y/o mentalmente, causando efectos negativos en la otra persona. En su sentido más amplio, la discriminación es una manera de ordenar y clasificar otras entidades. Puede referirse a cualquier ámbito y utilizar cualquier criterio. 
La discriminación de género adopta diversas formas de división de poder, algunos de cuyos aspectos incluyen: derechos humanos, prejuicios del rol masculino dentro de la casa, en el cuidado de los niños y en el trabajo. Aunque se calcula que las mujeres realizan dos terceras partes del trabajo en el mundo, las mujeres sólo obtienen una tercera parte de los ingresos y poseen menos del $1 \%$ de la propiedad mundial.

En nuestra investigación, hemos encontrado que el $65 \%$ de mujeres acep$\tan$ haber sido discriminadas frente a los varones. El otro $35 \%$ afirma que no y que tienen iguales derechos y forma de vida que los varones. Preguntadas cómo es que sienten esa discriminación, todas afirman que es en relación a la libertad para sus actividades que el varón tiene y la mujer no. Al otro grupo de "no discriminadas", se les repreguntó si tenían libertad para salir de su casa o para no cocinar ese día, o disponer de la totalidad de su tiempo o del dinero de la casa respondieron que no tenían ese dominio.

El 90\% de las mujeres opinaron que, por ser "más débiles", no llegan a obtener los mismos trabajos a los que accede el varón. Una mujer (10\%) cree tener las mismas posibilidades que el varón para trabajar: "sí podría trabajar en un trabajo de hombres, porque yo trabajo en todo..."; sin embargo, no cree que le puedan pagar el mismo salario, porque "siempre a los hombres en los trabajos les pagan más salario que a las mujeres...”.

Las mujeres consultadas (70\%) recuerdan que en su infancia fueron postergadas por sus padres a favor de sus hermanos varones: en cariño, compra de ropa, juguetes, permisos, juegos, etc. La discriminación de género es un fenómeno social, puesto que son necesarias representaciones de ambos sexos para que pueda darse esta situación; no existe una igualdad de género a partir de la cual denunciar la discriminación o desigualdad. Al contrario, la base de este fenómeno es la supuesta supremacía de uno de los géneros. ${ }^{20}$

La discriminación por género limita el potencial para el desarrollo. Las economías que achican la brecha entre los géneros y mejoran la posición de la mujer crecen más rápido, de acuerdo a un informe del Banco Mundial.

La violencia de género tiene que ver con "la violencia que se ejerce hacia las mujeres por el hecho de serlo", incluye tanto malos tratos de la pareja, como agresiones físicas o sexuales de extraños, mutilación genital, infanticidios femeninos, etc. ${ }^{18}$ y que pueda tener como resultado un daño o sufrimiento físico, sexual o psicológico para la mujer, inclusive las amenazas de tales actos, la coacción o la privación arbitraria de la libertad, tanto si se producen en la vida públi- 
ca como privada (Artículo 1 de la Declaración sobre la Eliminación de la Violencia contra la Mujer. Naciones Unidas, 1994).

El $80 \%$ de las mujeres entrevistadas reconocen haber sido maltratadas, amenazadas o golpeadas, durante su matrimonio o convivencia. El $60 \%$ reconoce como "normal" que así ocurra, porque el hombre es autoritario y es el "...jefe de la casa y da la manutención...". También pueden desarrollar los síntomas del trastorno de estrés postraumático, sentimientos depresivos, de rabia, baja autoestima, culpa y rencor; y suelen presentar problemas somáticos, disfunciones sexuales, conductas adictivas y dificultades en sus relaciones personales.

Y una tercera categoría, que pareciera asomarse también como fundamental dentro el estudio, es en lo que respecta a la influencia de la cultura y la sociedad, en la que se desenvuelven los seres humanos. La biología no define por sí misma el destino: los roles sociales y las conductas dejan su marca en la sexualidad de cada uno. Básicamente, se afirma que el género tiene que ver con los aspectos culturales con los cuales se interpreta la sexualidad.

Las tecnologías de la información y la comunicación (TIC) forman parte de la cultura que nos rodea; en casi todas nuestras actuaciones están presentes y debemos convivir con ellas, ya que amplían nuestras capacidades físicas y mentales y las posibilidades de desarrollo social. Pero, esta herramienta también tiene impactos negativos, como: el aislamiento, el fraude o menores puestos de trabajo. Las TIC también influyen en las personas y en la opinión pública. ${ }^{30,31}$

Las encuestas para esta investigación indican que en el 100\% de hogares, de todos los NSE A,B,D,E consultados, por lo menos tienen un televisor, y que en el $45 \%$ de los casos poseen una computadora, y que el $80 \%$ de hogares tienen por lo menos a un familiar que consulta con cierta frecuencia Internet. Otro instrumento que aparece como influyente en los jóvenes es el celular o teléfono móvil, especialmente el que posee tecnología para comunicarse con las redes sociales.

\section{Influencia sociocultural positiva y negativamente de los valores en la construcción de la sexualidad de los niños, jóvenes y adultos}

El 70\% de los entrevistados relata que los valores se están perdiendo entre los jóvenes, sobre todo en la relación padres e hijos, y el $80 \%$ expresa que los valores que más se han perdido son los de la familia. ${ }^{32}$

El 65\% de mujeres y hombres cree que las mujeres están relegadas en relación con los varones en la sociedad. Y entre las mujeres, esta percepción de la mujer relegada en la sociedad se expresa en el $90 \%$ de las entrevistadas. Los 
motivos, al parecer, son el patriarcado y machismo predominantes, la discriminación hacia la mujer que ejerce la sociedad en cuanto a trabajo, sueldos, género, en el hogar, etc. El 70\% de mujeres considera que actualmente las jóvenes tienen mayor libertad en el hogar, en el colegio y en lo sexual, piensan que "los varones han retrocedido en el gobierno del hogar".

Los resultados ponen de manifiesto la necesidad de establecer modelos complejos de educación sexual y campañas de información acerca de la sexualidad masculina y femenina. Como se puede ver, casi todos los participantes, muestran desconocimiento sobre su sexualidad, lo que probablemente está produciendo desajustes psicológicos, funcionales, sociales, de pareja y de convivencia ciudadana, lo cual impide explicar la satisfacción sexual y su relación con el bienestar físico y mental de las personas. Para el bienestar psicológico y subjetivo, las variables que mayor se explicaron fueron la satisfacción sexual y el atractivo físico y sexual percibidos.

\section{CONCLUSIONES}

La historia de la humanidad ha cambiado mucho la concepción de la sexualidad humana. El enfoque ha variado, fundamentalmente, desde la perspectiva social, de acuerdo con la aceptación o no que cada cultura tiene de la aproximación que cada persona posee (o debe tener, según los cánones socialmente aceptados) hacia su propia sexualidad. La falta de comunicación en la pareja es frecuente, factor de desacuerdos y violencia basada en género.

Las experiencias reales de la sexualidad de las niñas y las mujeres adultas están determinadas por una serie de permisos y restricciones impuestas por padres, escuelas y contexto social, que rara vez son aplicados por igual a los niños y los hombres. Al hombre se le permite ser infiel, asumiendo, de manea tácita, que es algo natural o biológico en el sexo masculino.

En relación al ejercicio de los valores dentro de la sociedad, ésta establece reglas morales para las personas en las relaciones sociales; así como hay una escala de valores morales, también la hay de valores inmorales o antivalores. Casi todos los participantes, de alguna manera muestran desconocimiento sobre su sexualidad, lo que probablemente está produciendo desajustes psicológicos, funcionales, sociales de pareja y de convivencia ciudadana. 


\section{REFERENCIAS BIBLIOGRÁFICAS}

1 Alvarez-GAYOU, J. 1999. La sexualidad humana como construcción multidisciplinar. Universidad de La Laguna, Tenerife.

2 AMNESTY INTERNATIONAL. 1994. Breaking the silence: Human rights violations based on sexual orientation. Amnesty International. New York,

3 Ávila, M B., 2001. Textos e imagens do feminismo: Mulheres construindo a igualdade. Universidade do Recife, Brasil.

4 Boff, L., Muraro, R.M. 1998. Femenino y masculino: el comienzo del fin del patriarcado. Interamericana. México.

5 Bott S, Morrison A, Ellsberg M. 2005. Cómo abordar la violencia de género en América Latina y el Caribe: revisión crítica de las intervenciones. OPS. México.

6 Burín, M, MELler L. 1998. Varones, género y subjetividad masculina. Paidos. Buenos Aires

7 ORganizACiÓn PANAMERICANA DE La SAlud. 2007. Violencia basada en género en América Latina. OPS. México.

8 CERRUTI, S. 1993. Salud y sexualidad desde una perspectiva de género. Revista Estudos Femenestas. México $\mathrm{N}^{\circ}$ 541:124-125.

9 Cerruti, S., Vega, L. 2008. Perspectiva histórica y concepción sobre la educación sexual. La travesía Uruguaya hacia la educación sexual. Altablero. Montevideo.

10 ORgANIZACIÓN DE LAS NACIONES UNIDAS 2010. Declaración sobre la Eliminación de la Violencia contra la Mujer. ONU, Colarebo. Brasil

11 De Los Ríos, R. 1993. Género, salud y desarrollo. Un Enfoque en la construcción. Género, Mujer y Salud en las Américas. México.

12 Engels, F., MARX, K. 1984. La Sagrada Familia. Deutschland. Berlin.

13 Ferreira, M. 2004: Género e políticas públicas. Revista Estudos Femenistas. 12 (1) 360: 48, 49. México.

14 Foucault, M.L 2000. Historia de la sexualidad, Tomo I: La voluntad del saber. Siglo veintiuno S.A.. Madrid.

15 Foucault, M. 1984. Historia de la sexualidad. Tomo II: El uso de los placeres. Siglo veintiuno, S.A., Madrid.

16 Hernández, S., Fernández, C., BAutista, P. 1998. Metodología de la Investigación. 2ed. Mc Graw Hill. México.

17 La Coste, C., Martorell, A. 2007. Las madres contra las mujeres: Patriarcado y maternidad en el Mundo. Ciencia Social Árabe. Argel.

18 LAFOSSE, V. S. 1987. Sociología de la familia peruana. Universidad Nacional José Faustino Sánchez Carrión. Educación a Distancia. Huacho, Perú.

19 LORA, C., BARNECHEA C. 1985. Reflexiones sobre sexualidad e identidad de la mujer. Shupihui. Vol. X N ${ }^{\circ}$ 35: 357-359. CETA Iquitos. Perú. ,

20 Lowy, M. 1996. Ideología y ciencia social. Universidade do Sao Paulo. Sao Paulo.

21 MACEY, D. 1995. Las vidas de Michel Foucault. Ediciones Cátedra, Madrid.

22 Matamala, M.I. et al. 1995. Calidad de la atención, género. Salud reproductiva de las mujeres. Comusams-Achnu. Santiago de Chile.

23 Ministerio De SAlud (MinsA). 2004. Plan General de la Estrategia Sanitaria Nacional de Salud Sexual y Salud Reproductiva. MINSA. Lima.

24 Ministerio De SAlud (MinSA). 2004. Guías Nacionales de Atención Integral de Salud Sexual y Reproductiva. Gráfica Ñañez. Lima. 
25 MoncriefF, H. 2007. Sexualidad y sociedad moderna. Revista de Filosofía. Vol.12: 203-205. Universidad de Medellín. Colombia.

26 ORTIZ, A. 1989. Apuntes para una lectura de Historia de la Sexualidad, tomo I, La voluntad de saber. Interamericana, Buenos Aires.

27 Murcia, N, Jaramillo, L. 2008. Investigación cualitativa. La Complementariedad. Kinesis, Caldas, Colombia.

28 Merlino, A, et al. 2009. Investigación cualitativa en ciencias sociales, América Lee. Buenos Aires.

29 KEE, J. 2005. Wome's human rights: violence against women, pornography and TICs. APC. Whasington. WNSP.

30 Malmotra, N. 2007. The world wide web of desire-content regulation on the internet. APC Washington. WNSP.

31 ISPA, UK. 2007. ISPA response to the European Commission Public Consultation on Safer Internet and online technologies for children. ISPA. London.

32 CRISTI, Cou. 2009. Valores humanos. (www.monografias.com/.../valores.../valoreshumanos.shtml. Consultado el 14 de Enero de 2013).

33 Dos Anjos, M. 2010. Valores humanos en sociedad. Consultado el 14 de Enero de 2013. (http://www.mercaba.org//juventud_y_crisis_de_valores_mor.htm). 